\title{
Autoimmune lymphoproliferative syndromes: genetic defects of apoptosis pathways
}

\author{
F Rieux-Laucat ${ }^{\star},{ }^{1}$, F Le Deist ${ }^{1}$ and A Fischer ${ }^{1}$ \\ 1 Hôpital Necker-INSERM U429, Paris Cedex 15, France \\ * Corresponding author: F Rieux-Laucat, Hôpital Necker, INSERM U429, 149 \\ rue de Sèvres 75743, Paris Cedex 15, France. Tel: +33-1-44-49-50-82; \\ Fax: +33-1-42-73-06-40; E-mail: rieux@ @ecker.fr
}

Received 23.4.02; revised 12.11.02; accepted 13.11 .02 Edited by G Melino

\begin{abstract}
Human and mouse natural mutants presenting with lymphoproliferative syndrome and autoimmunity (ALPS) have enlightened the role of the Fas and FasL in lymphocyte cell death and peripheral tolerance. Further study of the genetic basis of the human pathology led to the identification of apoptosis signaling defect, and pointed out to the crucial role of caspase-10 in the process of apoptosis induction. In contrast, the absence of lymproliferation in engineered mutants of 'death pathways' suggests that additional events are necessary to recapitulate the overt phenotype of ALPS patients or MRL/lpr mice. Moreover, these models highlight the roles of Fas and associated molecules, such as FADD and caspase-8, in lymphocyte development or activation. This review will discuss the main findings provided by the study of mouse models and human conditions.

Cell Death and Differentiation (2003) 10, 124-133. doi:10.1038/ sj.cdd. 4401190
\end{abstract}

Keywords: ALPS; death receptors; Fas; caspases

Abbreviations: ALPS, autoimmune lymphoproliferative syndrome; AICD, activation-induced cell death; DD, death domain; $D E D$, death effector domain; DISC, death-inducing signaling complex; ICD, intracytoplasmic domain; ECD, extracytoplasmic domain; DN, double negative; TCR, T-cell receptor; Ig, immunoglobulin; mAb, monoclonal antibody

\section{Death Receptors and Signalization of Apoptosis}

Apoptosis can be triggered by interactions of specialized membrane-bound receptors with their cognate ligands. These receptors, termed 'death receptors', belong to a TNF-receptor (TNF-R)/NGF-receptor (NGF-R) subfamily as they all contain cystein-rich domains (CRDs) in their extracytoplasmic region, and are characterized by the presence of a functional domain termed 'death domain' (DD) within the cytoplasmic region. ${ }^{1}$ Fas (also known as CD95 or Apo-1 and now as TNFRSF6) is a prototypical member with three extracellular CRDs and an 80 amino-acid residues intracellular DD. ${ }^{2}$

Five other receptors with DD can be found on peripheral blood mononuclear cells, TNF-R1, TRAMP (TNF-receptorrelated apoptosis mediating protein; DR3/ws/1/APO-3/ LARD), TRAIL (TNF-related apoptosis-inducing ligand)-R1 (DR4/APO-2), TRAIL-R2 (DR5/Trick/Killer) and DR6. ${ }^{2}$ The ligands that activate these receptors, with the exception of the DR6 ligand that remains unknown, are structurally related molecules that belong to the TNF superfamily. Fas ligand (FasL; CD95L) binds to Fas; TNF and lymphotoxin $\beta$ bind to TNFR1; APO3-ligand (APO-3L; TWEAK) and TL1-A binds to DR3; and TRAIL (APO-2 ligand, APO-2L) binds to DR4 and DR5. ${ }^{2}$ Several decoy receptors are described but their in vivo function remains a matter of debate. Two of them lack a transmembrane domain and exist either as a soluble form, like DcR-3, decoy receptor of FasL or as a GPi-linked receptor, like DcR-1 (TRAIL-R3). DcR2 (TRAIL-R4) retains an incomplete DD making it unable to trigger apoptosis, yet having some signaling capabilities like NF-kB activation. ${ }^{2}$

The remarkable feature of Fas and other death receptors signaling is the recruitment of a signal transduction protein complex within minutes upon triggering by their cognate ligand or agonistic antibodies. ${ }^{3}$ The formation of this complex called death-inducing signaling complex (DISC) is mediated by the specific conformation of the intracellular DD. An adapter protein called FADD (Fas-associated DD; also named MORT-1) then binds through its own DD to the clustered receptors' DD in its amino-terminal portion. FADD possesses another domain called death effector domain (DED), allowing interaction with an analogous domain repeated in tandem within procaspase- 8 and -10 (also called Flice/MACH-1 and Flice-2, respectively). ${ }^{3}$ It was previously shown that procaspase-8 was autoprocessed following its recruitment to the DISC via interactions with FADD.

Caspases are cysteine-proteases that cleave substrates after a specific aspartic residue. All caspases are synthesized as inactive proenzymes made of an amino-terminal caspase recruiting domain (CARD) and a large (p20) and a small (p10) protease subunit. DEDs are specific examples of the homophilic interaction domains called CARDs. Processing of caspases results in proteolytic cleavage of the zymogene form and association of the small and large protease domain in a heterotetramer complex forming the active caspase. ${ }^{4}$ Activated caspase- 8 and -10 can, in turn, activate other procaspases such as caspase-3, thereby initiating a proteolytic cascade that culminates to apoptosis. Several other cytoplasmic proteins were shown to interact with Fas such as Daxx, ${ }^{5}$ Rip $^{6}$ and FAF. ${ }^{7}$ However, their role in Fas-induced apoptosis remains to be discussed and may be different regarding cell types or stages of differentiation. 
The understanding of mechanisms underlying apoptosis induction upon triggering of Fas by its cognate ligand was recently revised by the work of Lenardo's group. ${ }^{8}$ Previously, the 'postligand' model proposed that trimerization of Fas, and thus signaling of apoptosis, was triggered by interaction with trimeric FasL. But Siegel et al., by using FRET technology, elegantly demonstrated that Fas molecules were previously trimerized through interactions of an $\mathrm{N}$-terminal domain called preligand association domain (PLAD). From a mechanistic point of view this means that ligand-receptor interaction may allow formation of receptor superclusters (made of oligomerized trimeres). Therefore, the stochiometry potentially required to induce apoptosis implies the engagement of at least six Fas molecules or may be more (as observed by using crosslinked anti-Fas antibodies). An alternative (but not exclusive) explanation may be that the FasL/Fas interaction could induce a change of the DDs conformation enabling further interactions with FADD and other downstream components. This may be simply explained by cooperativity of DDs to self-associate and/or by the dissociation of a DD silencer (SODD) as described for TNFRI.

Finally, a family of viral proteins called viral flice inhibitory proteins (v-FLIPS) and their cellular counterpart c-Flip (also called I-Flice, Cash, Casper, or FLAME) contain a DED similar to the corresponding domain in capase-8 and -10 and FADD. These molecules are potent inhibitors of Fas-induced apoptosis by interacting with FADD and/or caspase-8, and may have an important role in the regulation of several death receptor-induced apoptotic events. ${ }^{9}$ Indeed, while resting naive T cells express little surface Fas, T-cell receptor (TCR) stimulation induces Fas expression and renders activated $T$ cells progressively sensitive to Fas-induced apoptosis in the presence of IL-2. This increase in sensitivity has been correlated to a decrease in Flip that occurs after 3 days of T-cell activation in vitro. Besides its antiapoptotic activity, it has been proposed that c-Flip may also connect Fas signaling to costimulation through the ERK pathway and activation of NFkB. It was shown, a decade ago, that antibodies to Fas might provide costimulation for human $\mathrm{T}$ cells in vitro. This observation is supported by more recent studies showing that caspase-3 or - 8 inhibitors can inhibit anti-CD3 T-cell proliferation. ${ }^{10}$ However, the physiological significance of these observations remains poorly understood since Fas deficiencies in patients or in Ipr mice do not lead to immunodeficiency but rather to uncontrolled lymphoproliferation (see below).

\section{Features of Lymphoproliferative Syndrome with Autoimmunity (ALPS)}

More than 30 years ago, Canale and Smith as well as others reported a condition characterized by nonmalignant lymphadenopathies associated with autoimmune features in children. ${ }^{11}$ However, the genetic basis of this lymphoproliferation with autoimmunity was firstly identified in MRL Iprmice. ${ }^{12}$ This natural mouse mutant strain was considered as a model of human lupus since these mice develop nephritis, hypergammaglobulinemia and antinuclear antibodies in addition to lymphadenopathy. With age, these mice accumulate CD $4^{-} \mathrm{CD}^{-}{ }^{-} \mathrm{TCR} \alpha \beta^{+}$T cells in the periphery. This phenotype is likely the consequence of the downregulation of CD8 expression on mature peripheral lymphocytes. The autoimmune features vary from strains to strains carrying the $/ p r$ mutation. Other natural mutants were subsequently described, that is, the $l p r^{c g}$ and gld mice. ${ }^{13}$ While the $l p r$ strain is characterized by an almost complete defect of Fas expression, the $I p r^{c g}$ mutation allows the expression of a nonfunctional protein. In the gld mouse, a missense mutation in the extracellular domain (ECD) of FasL abrogates its function. The phenotype of these mice is very similar, with variable time course of symptoms onset, the shortest one being observed in the Fas-knockout model that represents a unique model of complete Fas deficiency. ${ }^{14}$

By analogy to the animal model, defects of the Fas pathway were identified in patients few years ago. ${ }^{15-17}$ The syndrome was also named lymphoproliferative syndrome with autoimmunity. ${ }^{15}$

In humans, ALPS is defined by functional analysis of lymphocyte sensitivity to Fas-induced apoptosis in vitro. T cells from ALPS patients can exhibit complete Fas-induced apoptosis (ALPS 0), consequence of a complete expression deficiency, partial Fas-induced apoptosis defect associated with normal or slightly reduced Fas expression (ALPS I and II), and finally normal Fas-induced apoptosis (ALPS III). Phenotype and genetic basis of these functional groups are diverse (Table 1). Nevertheless, as discussed below, four criteria characterize the ALPS condition: tumoral syndrome (splenomegaly and/or lymphadenopathies), autoimmune manifestations, hypergammaglobulinemia and detection of $\operatorname{TCR} \alpha \beta$ $\mathrm{CD}^{-}{ }^{-} \mathrm{CD} 8^{-}$T cells (hereafter called double negative or DN) in blood. Besides few exceptions, ALPS patients are presenting with at least three of them.

Table 1

\begin{tabular}{|c|c|c|c|c|}
\hline ALPS & Genotype & $\begin{array}{l}\text { Phenotype onset/ } \\
\text { severity }\end{array}$ & Death-receptor defect & Mouse model \\
\hline 0 & Homozygous Fas mutations & Prenatal/severe & $\begin{array}{l}\text { Complete Fas expression and } \\
\text { function defect }\end{array}$ & Ipr/lpr, Fas ko \\
\hline la & Heterozygous Fas mutations & Childhood/moderate-severe, Al & Partial Fas function defect & $I p r^{c g} / / p r^{c g}$ \\
\hline $\mathrm{Ib}$ & Heterozygous FasL mutations & Adulthood/SLE & None & gld/gld \\
\hline II & Caspase-10 mutations & Childhood/moderate, $\mathrm{Al}$ & $\begin{array}{l}\text { Partial Fas, TNF RI, Trail R 1, 2, DR3. } \\
\text { Function defect }\end{array}$ & Unknown \\
\hline III & $?$ & Childhood/moderate, $\mathrm{Al}$ & Unknown & Unknown \\
\hline
\end{tabular}

Al: autoimmunity; SLE: systemic lupus erythematous. 


\section{Complete Fas deficiency: ALPS 0}

The first complete defect of Fas expression was identified from a patient born to consanguineous parents. ${ }^{15,18}$ The child had massive lymphoproliferation evident at birth indicating a process that had started in the prenatal period. This is different from both $I p r$ and Fas-knockout mice. Lymphoproliferation involved both $T$ and $B$ cells and was associated with mild autoimmunity causing thrombocytopenia. Lymphocytes from this patient were insensitive to treatment by an agonist antiFas antibody. Some characteristics of the T/B lymphoproliferation in this child deserve further comments. More than $70 \%$ of the peripheral T cells had a DN phenotype (TCR $\alpha \beta$ $\mathrm{CD} 4^{-} \mathrm{CD} 8^{-}$). Numerous mitotic figures were observed in spleen and lymph node sections, and suggested active lymphocyte division rather than passive lymphocyte accumulation. The rapid rise in circulating lymphocyte counts, seen following chemotherapy treatment, also supports this hypothesis. In the absence of Fas expression on peripheral lymphocytes, an early onset (prenatal) activation, proliferation and therefore accumulation of autoreactive $T$ and $B$ cells is assumed. Finally, it was striking to observe lymphocytes undergoing apoptosis on blood smears as well as in the spleen sinuses. Such a spontaneous apoptosis is also observed in culture, as $90 \%$ of the T cells are dying within $24 \mathrm{~h}$ ( $\mathrm{F}$ Le Deist et al., unpublished observations). These results suggest that an alternative pathway(s) of lymphocyte apoptosis is engaged in an attempt to compensate for the Fas deficiency. In addition, this human complete Fas deficiency has been found to be curable by allogeneic bone marrow transplantation, ${ }^{19}$ indicating that consequences of the Fas deficiency was restricted to lymphocytes or at least to cells of hematopoietic origin.

Two other cases of complete Fas deficiency have been since described. ${ }^{20,21}$ Outcome of the phenotype was strikingly similar, with prenatal onset, very severe lymphoproliferation accompanied by lung infiltration and leading to death in one case. $^{21}$

\section{Partial Fas deficiency (ALPS la and II)}

Two types of defect can potentially underlie this deficiency: an intrinsic Fas defect as in ALPS la or a defect in the signaling pathway as in ALPS II. While the former is the main cause of ALPS, the latter has been only described in two pedigrees. A common finding in all these patients is a defective Fas-mediated apoptosis of T (and B) lymphocytes, whereas a normal or slightly reduced expression of Fas is detected on activated T cells. ${ }^{18,22}$ The overall phenotype is similar in both types. Nevertheless some differences were noted. Given the very few cases of ALPS II, it is difficult to conclude whether the observed phenotype is related to the molecular basis of the disease or whether it is a particularity of these families. The salient features of ALPS II are discussed together with the genetic basis of this defect.

In ALPS la, the magnitude of the defect varies from one patient to another. In parallel, antigen-induced T-cell death (activation-induced cell death (AICD)) is also impaired. Of note, the magnitude of the defect does not correlate with the onset and severity of the clinical manifestations as individuals carrying the same Fas mutation, exhibiting the same Fasmediated apoptosis defect, could be symptomatic or not (see below). Onset of symptoms occurs in early childhood ( $0-5$ years, around 6-12 months in most cases). The lymphoproliferation is usually the most salient manifestation causing lymphadenopathies and hepato-splenomegaly (observed in $80 \%$ of patients). Lymphoproliferation consists in the accumulation of nonmalignant polyclonal $\mathrm{T}$ lymphocytes. A variable fraction of these lymphocytes (2-61\% of $\alpha \beta$-T cells) exhibit a typical DN phenotype. There is also excessive $B$ lymphocyte accumulation in some patients but not all. T cells as well as B cells accumulate in paracortical areas while the overall architecture of lymphoid organs is preserved. Many of those cells do express the Ki-67 antigen indicative of active proliferation. ${ }^{18}$ These features indicate that defective Fasmediated apoptosis of both $T$ and $B$ lymphocytes results in the accumulation of polyclonal cells that further proliferate. The autoimmune specificity of these lymphocytes is postulated. ALPS la condition is usually less severe than ALPS 0 , but rare cases of severe lymphoproliferation with prenatal onset were described in this setting. ${ }^{23}$

By a long-term follow-up of a number of patients, it has been possible to determine that there is a significant shrinkage of lymphoproliferation (down to complete regression of splenomegaly and adenopathies) in a number of them over time, even in the absence of any treatment. ${ }^{23}$ Surprisingly, hypergammaglobulinemia and DN T cells stay relatively constant and tend to persist throughout life. This is in major contrast with the evolution of the disease observed in /pror gld mice. It can be hypothesized either that the predominant influx of naive T and B cells in early childhood favorizes lymphocyte accumulation as compared to adults and/or that alternative apoptotic pathways are used by human lymphocytes later in life.

Autoimmune manifestations are the second most frequent event in patients with ALPS la (Table 2). In all, 42 out of the 56 reported patients have developed clinical symptoms associated with autoantibodies. Age at onset varies considerably

Table 2

$\begin{array}{ll}\text { ALPS la } & \begin{array}{l}\text { Autoimmune } \\ \text { manifestations }\end{array}\end{array}$

56 patients

Autoimmune hemolytic anemia $\quad 22$

Thrombopenia 19

Neutropenia

Skin (urticaria, vasculitis)

Glomerulonephritis

Guillain Barre syndrome

Iridocyclitis/uveitis

Arthritis

Hepatitis

Autoimmune biliary cirrhosis

Seizures ${ }^{a}$

Ovarian failure $^{a}$

Mucosal ulcerations ${ }^{a}$

19

10

4

3

2

2

1

${ }^{a}$ Autoimmunity suspected. 
(in contrast to the lymphoproliferative syndrome). ${ }^{18,20,23-28}$ It is therefore possible that among reported patients, some who have not yet developed such manifestations, will do it later on. A striking observation is that autoimmunity appears to be always associated with autoantibodies, although direct pathogenic intervention of $T$ cells in some of the autoimmune processes cannot be excluded. In none of the patients typical systemic lupus erythematous was detected (none had anti-DNA antibodies). In some of the patients, autoimmunity tends to be severe, requiring aggressive immunosuppressive regimens (including steroids and cyclophosphamide), in order to be controlled. Splenectomy, often performed because of discomfort and hypersplenism, was also, in some cases, required because of protracted autoimmunity toward blood cells. Typical organ-associated autoimmunity is rare but can be found in some instances such as autoimmune hepatitis ${ }^{29}$ or even diabetes. ${ }^{30}$

Failure to thrive is a frequent symptom in children. Of note, splenectomy could reverse it in a number of cases. Malignant diseases have been reported, that is, lymphomas, liver carcinomas (with hepatitis $\mathrm{C}$ infection) and severe thyroid and breast adenomas together with basal cell carcinomas. ${ }^{16,17,31}$ These malignancies occurred after the age of 15 years. Therefore, it appears that Fas mutations represent a significant risk factor for malignancy, especially for Hodgkin's lymphomas $^{32}$ and to a lesser extent for non-Hodgkin's lymphomas. $^{33}$ Descriptions of somatic Fas mutations in germinal centers, as consequences of the process of $\mathrm{Ig}$ somatic mutations, and in leukemia and lymphoma support this hypothesis. ${ }^{34,35}$

Unusual T cells, that is, TCR $\alpha^{+} \beta^{+} \mathrm{CD}^{-}, \mathrm{CD}^{-}, \mathrm{CD}^{-} 5 \mathrm{RA}{ }^{+}$, $\mathrm{CD}^{2} 7^{+} \mathrm{T}$ cells are detected in excess in the blood of most patients with ALPS la, and a rise of this population is frequently observed after splenectomy. This subset accounts for $2-60 \%$ of the blood T-cell counts, instead of $<2 \%$ in controls. The lymphocyte counts are variably increased, reflecting the intensity of the lymphoproliferative syndrome. Interestingly, a slight increase of these cells in the blood of healthy relatives of patients was recently described. ${ }^{36}$ However, it is not clear yet whether these cells play a role in the physiopathology of the disease. Experiments carried out with $\mathrm{T}$ cells of $I p r$ mice have determined that $\mathrm{CD}^{-} \mathrm{CD}^{-} \mathrm{T}$ cells mainly derived from $\mathrm{CD}^{+} \mathrm{T}$ cells and are in an anergic status, unable to be activated by antigens and poorly by mitogens. ${ }^{13}$ Much less information is available about human DN T cells origin. In contrast to their mouse counterparts, human DN T cells in ALPS are CD2 ${ }^{+}$. They strongly express FasL and IL-10. ${ }^{37}$.

A striking feature of ALPS la consists in the overproduction of IL-10 as well as reduced IL-12 production by monocytes and increased IL-10 plasma level. It can be postulated to be a secondary regulatory event attempting to counterbalance the persistence and activation of autoimmune clones. Also, T cells have mostly a Th2 phenotype. ${ }^{38}$ Polyclonal hypergammaglobulinemia $G$ and $A$ is a very frequent finding while the level of serum IgM is usually reduced. Findings in mouse models suggest that $\mathrm{B}$-cell proliferation is driven by $\mathrm{T}$ cells and strongly associated with the proliferation of either CD8 or DN T cells. $^{12}$

\section{Absence of Fas-induced apoptosis defect (ALPS Ib and III)}

Routinely, the sensitivity to Fas is assessed using monoclonal antibodies against the receptor. Therefore a defect of FasL or of another death receptor would give rise to a normal functional assay. A unique case of FasL deficiency has been described so far. ${ }^{39}$ However, the phenotype of this case is substantially different from the classical ALPS (see molecular description below). In more than 30 patients with ALPS we cannot ascribe any functional defect. Patients lymphocytes exhibit a normal Fas/FasL pathway in spite of a typical ALPS phenotype. Other death receptor functions are currently under investigation.

\section{Genetic and Molecular Basis of ALPS}

The recognition that Ipr mice carry a mutation of the Fas gene was a major advance in the understanding of the role of the Fas molecule. ${ }^{12}$ It has also pointed the way to the identification of the human counterpart. Ipr mutant mice have an insertion of a transposon into intron 2 of the Fas gene that dramatically reduces the normal splicing of the Fas transcript. A similar, albeit less severe condition, $I p r^{\mathrm{cg}}$, is associated with a missense mutation within the so-called 'DD' encoding part of the Fas gene. Mutations of the FasL gene also result in lymphoproliferation.

Similarly, the human pathology is also diverse as at least five types can be described (see Table 1).

\section{ALPS 0}

ALPS caused by complete Fas deficiency (ALPS 0) are consequences of homozygous null mutations. Three cases of homozygous mutations were reported. ${ }^{15,20,21}$ In one case, a stop codon in the ECD was predicted to lead to a complete expression defect. In contrast, in the two other cases mutations are localized in the intracytoplasmic domain (ICD), affecting the DD-encoding exon 9. These mutants should be expressed at the surface, according to truncation experiments performed on human Fas expressed in murine fibroblasts. ${ }^{40}$ The complete expression defect of these mutants can thus result from either instability and premature elimination, or retention in the ER (because of the neo-peptide encoded by the mutations). Since such mutations were inherited from healthy parents (carrying the mutation at the heterozygous state), it was proposed that these mutations were recessive. ${ }^{21}$ However, another unpublished case of family with ALPS 0 patient is not in support with this conclusion. In that kindred, whereas the mother is healthy and carries the heterozygous mutation, the father, with the same genotype, presented with symptoms of classical ALPS la (Rieux-Laucat et al., unpublished observations). Importantly, cells from both parents exhibited a Fas-induced apoptosis defect of the same magnitude. The child, who received both mutated alleles, is presenting with complete Fas deficiency and typical ALPS 0 . From this example, it can be suggested that most, if not all, mutations are dominant, and that when homozygous, they lead to a more severe 
phenotype. This is a classical observation in dominant diseases.

In one family, patients are compound heterozygotes, with one mutation resulting in an amino-acid substitution in the ECD. ${ }^{41}$ It is unclear whether this modified Fas molecule has an impaired function. These patients exhibit a phenotype similar to the one of ALPS la.

\section{ALPS la}

More than 60 patients carrying a heterozygous Fas mutation have been so far reported in the literature. ${ }^{18,20,21,23-}$ $28,30,33,36,41-47$. A variety of Fas gene mutations have been found associated with ALPS la including mutations leading to truncated products or modified sequences (Figures 1 and 2). The modified Fas molecules exert a transdominant negative effect on Fas-mediated apoptosis as shown by transfection experiments. ${ }^{27,48}$ Most of the identified mutations deal with the intracellular domain. In all, $60 \%$ are localized within the DD. Approximately half results in truncated products and the second half in Fas molecules with a modified sequence at one position. Given that mutated protein can be detected at the membrane level (F Rieux-Laucat et al., unpublished observations), random incorporation into Fas trimers of abnormal molecules likely account for it. These mutants result in reduced FADD binding and caspase recruitment much greater than a $50 \%$ reduction as predicted in a $1: 1$ noncooperative interaction between Fas and FADD. ${ }^{48}$ This is therefore suggesting that there is cooperativity between Fas subunits in the recruitment of FADD, consistent with the presence of only one out of eight normal trimers in this setting.

One-third of mutations affect the ECD of Fas. They are usually associated with a less important Fas-induced apoptosis defect as compared with ICD mutations. ECD mutations either result in truncated products unable to anchor the membrane or expression of an abnormal Fas molecule, most likely incapable of interaction with FasL. The former mutations should be considered as loss of function mutations and the associated defect can thus be explained by haplo-insuffi-

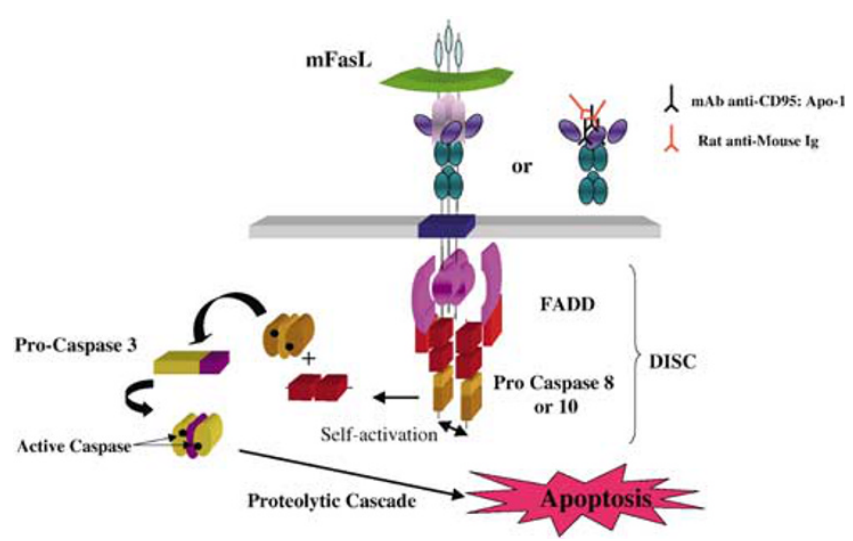

Figure 1 Fas-L/Fas induction of lymphocyte apoptosis. Upon interaction of membrane-bound FasL (mFasL) with Fas, or using a cross-linked Apo-1 (antiFas) monoclonal antibody (mAb), FADD, caspase- 8 and -10 are recruited into a molecular complex called DISC. Activated caspase-8 is in turn able to trigger a biochemical cascade leading to apoptosis ciency of the fas gene. However, such mutants exert a dominant negative effect on wild-type Fas when cotransfected in Fas negative cells. ${ }^{25}$ The PLAD model allows understanding how these mutants can affect triggering of apoptosis. Although unable to anchor the membrane, ECD mutants resulting from nonsense mutations can still be associated to the receptor complex through the $\mathrm{N}$-terminal domain. ${ }^{8}$ The identification of a mutation leading to a stop codon at position +12 (F Rieux-Laucat et al., unpublished observations) would restrict the PLAD to the signal peptide and the very first residues of Fas. Alternatively, it can be envisaged that such a short mutant would not be expressed, thus leading to haploinsufficiency. This last hypothesis is supported by experiments performed on thymocytes from heterozygous Fasknockout mice, which exhibit a reduced Fas-induced apoptosis. ${ }^{49}$ Further experiments are needed to distinguish between these two possibilities.

It is striking to note that Fas deficiency is observed in cells from all carriers of heterozygous mutations. Thus, on a functional standpoint, mutations are fully penetrant. In contrast, only $70 \%$ of the carriers of heterozygous Fas mutations develop clinical symptoms. ${ }^{23,25,27}$ This clinical penetrance is highest for ICD missense mutations, reaching $90 \%$. Mutations leading to ICD truncation have a clinical penetrance of roughly $75 \%$. Finally, the clinical penetrance dropped to $30 \%$ for ECD mutations. This difference is the only genotype-phenotype correlation that can be made so far. Otherwise no correlations were found between mutations, magnitude of Fas-induced apoptosis defect and severity of the syndrome.

This partial clinical penetrance strongly suggests that a second event should be associated with Fas mutations in order to induce an overt ALPS la syndrome. It is likely that genetic rather than environmental factors, like in Ipr mice, influence the ALPS expression, and account for variable penetrance of some of the mutations. So far, none of these gene modifiers have been identified. These gene products can be either directly involved in the apoptotic process, its regulation or in other events controlling activation, proliferation and survival of autoimmune clones. It is striking to note that mutations of both Fas alleles (either in homozygous or in compound heterozygous) are associated with full clinical penetrance. Similarly, in mice, symptoms develop only in homozygous animals, despite a potential defective Fasinduced apoptosis in heterozygous animals. More importantly, it was reported that double heterozygous $1 \mathrm{pr} / \mathrm{gld}$ animals develop a mild lymphoproliferative disease. ${ }^{50}$ These findings suggest that accumulation of defects in the Fas/FasL may lead to development of the syndrome. In contrast, the finding of a slight, but statistically significant, increase of DN T cells in parents who do not carry a Fas mutation (and therefore have normal Fas-induced apoptosis) implies that the second event may be independent of the apoptosis Fas-pathway. ${ }^{36}$

\section{ALPS Ib}

A unique case of dominant FasL mutation has been described in a patient presenting with features of systemic lupus erythematous along with chronic lymphoproliferation. ${ }^{39}$ It was defined as ALPS $\mathrm{lb}$ although the phenotype does not 


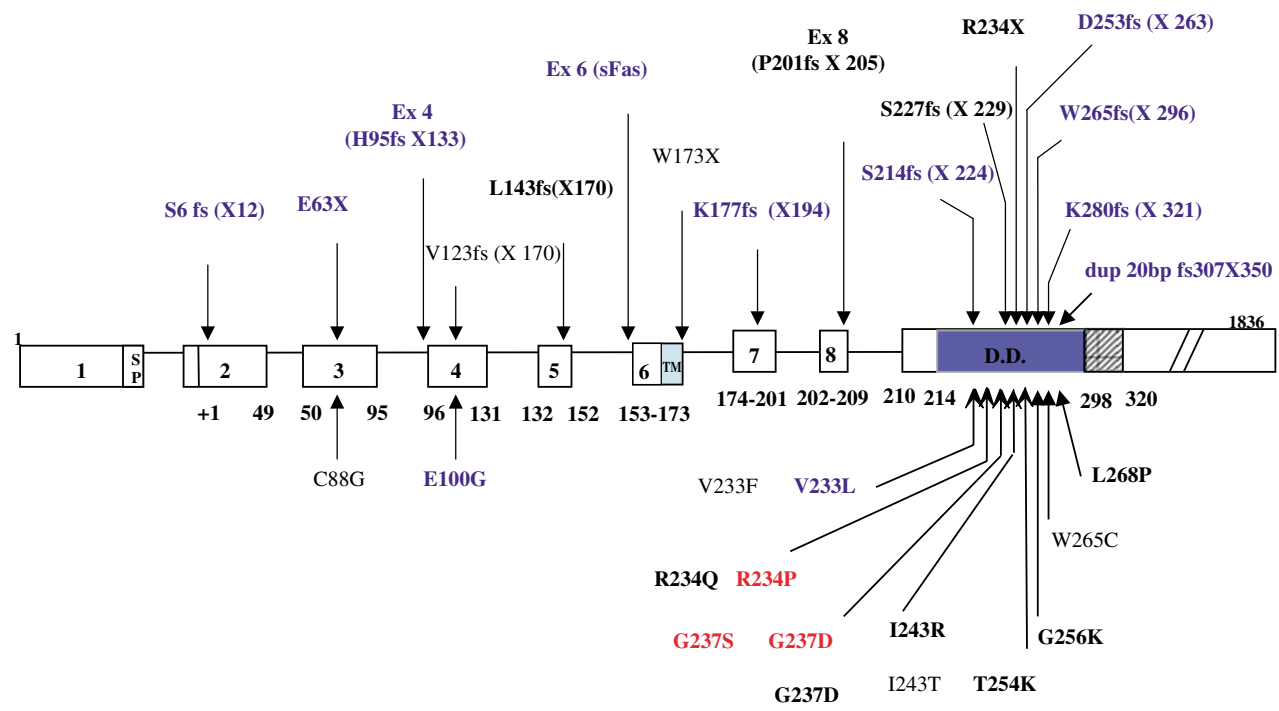

Figure 2 Heterozygous Fas mutations characterized at our center are shown. Most of them are localized into the intracellular DD of Fas. Mutations in red have full clinical penetrance (all carriers develop clinical manifestations) and those in blue partial clinical penetrance. (some individuals carrying a Fas mutation did not develop clinical symptoms despite an in vitro Fas-induced apoptosis defect). Mutations in bold black are de novo mutations (mutations in normal black are of unknown inheritance)

fulfill the criteria of classical ALPS (DN T cells and splenomegaly were absent). The clinical manifestation could represent a bias as the search was performed in a cohort of patients with SLE.

A search for FasL mutation in all ALPS 1a patients (as a potential second factor), as well as in more than 20 patients classified as ALPS III (see below), failed to identify any mutation. Two changes observed in the FasL cDNA turned out to be polymorphism since they did not affect the outcome of AICD in vitro (F Rieux-Laucat et al., unpublished observation). Given the similar phenotype of $I p r$ and gld mice, one could have anticipated an occurrence of FasL mutation in humans as frequent as Fas mutations. The absence of inherited FasL mutations in ALPS can be interpretated in several ways. On the one hand, one may speculate that FasL is more important for human development than it is in mice. Thus, a FasL defect would not be compatible with life. On the other hand, FasL defect might lead to a completely different phenotype, such as an immune deficiency if FasL is involved in triggering cell proliferation. Severe disease (related to potential extrahematopoietic manifestations) masking the diagnosis of ALPS is another possibility. Finally, an absence of phenotype would then suggest the existence of an unforeseen ligand in humans.

\section{ALPS II}

A number of patients are presenting with all typical clinical and immunological features of ALPS including abnormal lymphocyte Fas-mediated apoptosis. However, Fas molecule expression and sequence are normal in these patients. Although, until recently, the molecular basis of ALPS II was unknown, it is likely that the defect lies downstream of Fas in the cascade leading to cell apoptosis. Recently, Wang et al. ${ }^{22}$ have reported the occurrence in two families of caspase-10 mutations associated with ALPS. These findings are extremely important for several reasons. It is unexpectedly shown that caspase-10 plays a major role in Fas-mediated apoptosis. Mutated caspase-10 molecules impair autoprocessing of the caspase-10 molecules, and more importantly caspase- 8 activation while DISC recruitment is normal. It is therefore strongly suggested that, besides caspase-8, the related caspase-10 is also incorporated into the DISC upon Fas engagement. More recent works confirmed this finding and showed the key role of caspase-10 in Fas-induced apoptosis. ${ }^{51,52}$

A second interesting finding was that not only the Fasmediated apoptosis pathway is impaired but also apoptosis triggered by TNF R1, DR3 and Trail receptors DR4 and DR5. Caspase-10 appears thus involved in the apoptotic cascade of all known receptors inducing lymphocyte apoptosis. Strikingly, dendritic cells (DCs) accumulation was noted in lymphoid organs of caspase-10 but not of Fas-deficient patients. The authors speculate that the defective death of $\mathrm{T}$, B lymphocytes and DCs in this setting could account for a possibly more severe autoimmune phenotype observed in these patients. DC death has been shown to be Trail rather than FasL dependent. Caspase-10-deficient patients were indeed reported to exhibit extremely severe autoimmune hemolytic anemia and thrombocytopenia, and optic neuritis and meningitis, respectively. As it has been previously reported that DC death by apoptosis is involved in homeostasis of immune responses, ${ }^{53}$ abnormal persistence of DC might also contribute to the activation of autoimmune clones. This remains however to be demonstrated.

Interestingly, one of the mutations exerts a transdominant negative effect impairing Fas as well as other death receptormediated apoptosis, while the second requires expression on 
both alleles to induce a full-blown clinical picture. Detection of the latter mutation at the heterozygous state with a high frequency in the Danish population has disputed its involvement in the onset of ALPS II. Nevertheless, description of a healthy individual carrying this homozygous mutation/ polymorphism is still awaited. In any case, the dominant caspase-10 mutation has been validated and highlights the physiological role of this caspase in the cascade leading to apoptosis.

Other patients with ALPS II do not present caspase-10 deficiencies (F Rieux-Laucat et al., unpublished observations). Therefore, other known or yet unknown molecules involved in the Fas apoptotic pathway should be defective in these cases.

Of note is the lack of animal models equivalent to ALPS II. Importantly, a functional caspase-10 does not exist in mice. In addition, the caspase-8-knockout is embryonic lethal, implying a central role of this caspase and the corresponding apoptosis pathway in the regulation of development. Mouse embryonic fibroblasts derived from caspase-8-knockout embryos exhibit a block of Fas-, TNFRI- and DR3-induced apoptosis, confirming the key role of this caspase in the regulation of these pathways. Given this strong phenotype in mice one would not expect to see caspase-8 defects in humans. However, the recent description of a homozygous caspase-8 mutation in two siblings presenting with a mild immunodeficiency is strikingly different from the mouse model. ${ }^{54}$ These data are supporting an early and crucial role of caspase-8 in lymphocyte proliferation. As this is a unique observation, other cases are awaited in order to confirm these surprising findings. Other evidence linking Fas to proliferation pathways came from studies of FADD-deficient mice. Owing to the embryonic lethality of the FADD KO mice, FADDdeficient embryonic stem cells were used to complement RAG-I-deficient blastocysts. Expectedly, lymphocytes of chimeric mice had defective death receptor-induced apoptosis. But surprisingly, these mice exhibited B-cell depletion and impaired TCR-induced proliferation. ${ }^{55}$ Therefore, partial defects of FADD may also potentially lead to immunodeficiency rather than to ALPS.

Finally, transgenic animals expressing inhibitors of caspase-8, such as c-Flip and Crma were studied. Whereas all death-receptor pathway analyzed were blocked, neither lymphoproliferations nor immunodeficiencies were observed. Although these puzzling observations may reveal transgenic artifacts they suggest a new scheme for the role of these signaling molecules. Firstly, accumulating evidences involve FADD and caspase- 8 in lymphocyte proliferation. This may be directly after antigen-receptor stimulation and eventually independent of death receptors. Secondly, from ALPS II studies it turns out that caspase-10 may be specialized in apoptosis signalization. Such a specialized molecule remains to be identified in mice. Finally, one may speculate that different signaling complex may form depending on cell activation or differentiation allowing a same receptor to signal either proliferation or apoptosis. Further interaction analyses of FADD, caspase-8, caspase-10, Flip and potentially other unknown molecules are needed to resolve this issue and potentially to identify the modifier factors involved in ALPS.

\section{ALPS III}

We have investigated a number of patients (over 30) who present with a clinical condition close to mild ALPS, associated with hypergammaglobulinemia and excess of blood DN T cells (unpublished observation). Patients lymphocytes exhibit a normal activation of the FasL/Fas pathway. No molecular defects have been found so far. Although not demonstrated, it is plausible that in these patients, another lymphocyte apoptotic pathway is impaired. Recent descriptions of death-receptor deficiencies in knockout mouse models provide informations about their potential role in lymphocyte homeostasis. Analyses of DR6-deficient mice showed enhanced $\mathrm{CD} 4^{+} \mathrm{T}$-cell proliferation with a profound Th2 polarization. ${ }^{56}$ This phenotype appears independent of the apoptosis function of DR6 but rather implies connections with the c-Jun amino terminal kinase (JNK). Therefore, DR6 functions could be related to Th2 attenuation through activation of JNK. ${ }^{56,57}$ This is of interest for ALPS studies as a bias toward Th2 cytokine production was reported in some patients. ${ }^{10}$ Another interesting observation was made in DR3deficient animals. ${ }^{58}$ These mice exhibit negative selection impairment in the thymus when crossed to the HY-transgenic model. Whereas anti-CD3-induced apoptosis of thymocytes is defective, pre-T-cell checkpoint, positive selection and superantigen-induced negative selection are normal. This suggests that $T$ cells with moderate but significant selfreactivity may reach the periphery in these mice. One may therefore consider that one of these receptors or both can be involved in ALPS III. They can also be implicated in ALPS la as a second genetic event participating in the triggering of the syndrome. If these hypotheses are correct, then Fas-independent apoptotic pathway(s) is(are) important mediators of the control of lymphocyte homeostasis in humans. It would thus be important to determine which one.

\section{Mechanism of Autoimmunity in ALPS}

Autoimmunity is a key feature of ALPS as it is in Fas-deficient murine mutants. It is present in most patients and appears to be mostly antibody mediated. Therefore, the relation between defective FasL/Fas pathway and onset of autoimmunity is established. It appears that antigen-induced cell death of autoimmune clones is more profoundly affected than that of clones specific for exogenous antigens. There is indeed no obvious expansion of $\mathrm{T}+/-\mathrm{B}$ cell clones following infection or immunization. In contrast, transient reduction in size of lymphoid organs has been noticed in ALPS patients during an infection. ${ }^{59}$ This suggests that the FasL/Fas pathway is mostly involved in AICD during chronic exposure to autoantigens. Rathmell and Goodnow have proposed an elegant model to account for the role of the FasL/Fas pathway in the control of autoreactive T and B cells. ${ }^{60}$ Chronically stimulated $\mathrm{T}$ cells downmodulate CD28 and therefore become more vulnerable to FasL-induced cell death according to either a suicide or a killing mechanism. ${ }^{12}$ Fas-deficient T cells escape cell death, proliferate and can activate Fas-deficient B cells. In physiological conditions, FasL-expressing $T$ cells kill normal autoimmune B-cell clones, likely because chronic exposure to 
antigens no longer induce protecting signals from cell death. Fas-deficient $B$ cells will thus escape this regulatory process and further proliferate. CD40L/CD40 T/B interaction can induce class-switch and further affinity maturation of $\mathrm{Ig}$ with autoimmune specificity. B cells produce autoantibodies in $T$ cell zones where 'normal' autoimmune B cells, excluded from B-cell zones, are programmed to die. ${ }^{60}$

Therefore, FasL/Fas pathway deficiency creates a defect in peripheral tolerance. Central tolerance at least for $T$ cells does not appear to be impaired as shown in Ipr mice since negative selection normally occurs in Ipr mice transgenic for a given TCR. ${ }^{61}$ These results do not exclude a minor role of FasL/Fas in the thymus. Also, it would be interesting to know whether central tolerance is affected in the caspase-10 deficient setting.

Autoimmune features vary from strain to strain carrying the Ipr mutation. These manifestations are moderate in C57BL6 or 129 mice. $^{12}$ This important finding shows that Fas mutations are a predisposing factor to the onset of autoimmunity, but that the latter requires other genetic susceptibility factors. Loci encoding some of them have been recently mapped. ${ }^{62}$ Further genetic studies of mice should thus lead to the identification of other important gene products in the control of autoimmunity. Similarly in humans, autoimmune manifestations may be driven by a third genetic event. For instance, the observation that in one given family (Figures 3 and 4), among three people carrying the same mutation, that is, the father and two children, one individual developed the full-blown syndrome including lymphoproliferation and severe autoimmunity, a second one (who is older) lymphoproliferation but no autoimmune manifestations and a third (a parent) without any clinical manifestations suggest that there might be several modifier gene products.

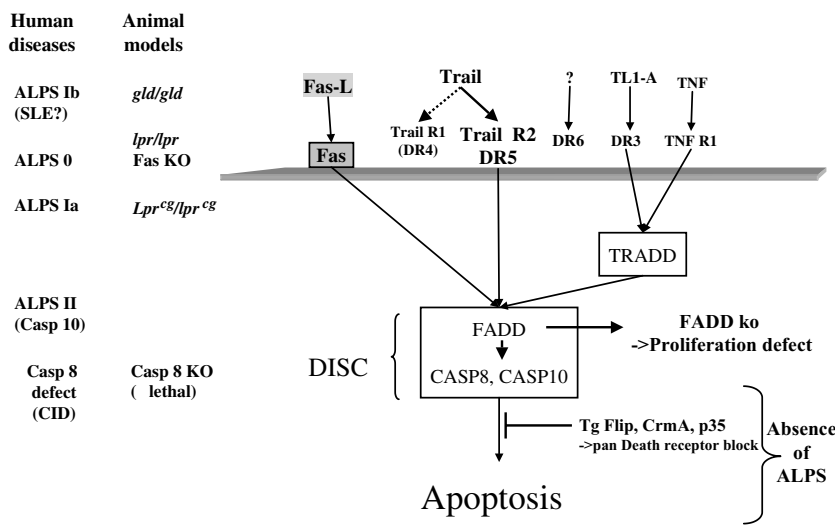

Figure 3 Summary of known death ligand/receptor signaling defects: corresponding human and mouse gene defects are aligned. ALPS $\mathrm{lb}$ is the human genetic counterpart of the gld mice, but the phenotype is different from other ALPS (see text). The human caspase-8 defect gives rise to a combined immunodeficiency (CID), whereas the mouse caspase-8 defect is embryonic lethal ( $\varepsilon$ lethal). The FADD-knockout is embryonic lethal, but when the defect is limited to lymphocytes it leads to a profound immune deficiency. Transgenic animals expressing an inhibitor of caspase-8 do not develop lymphoproliferation although all death-receptor pathways are blocked

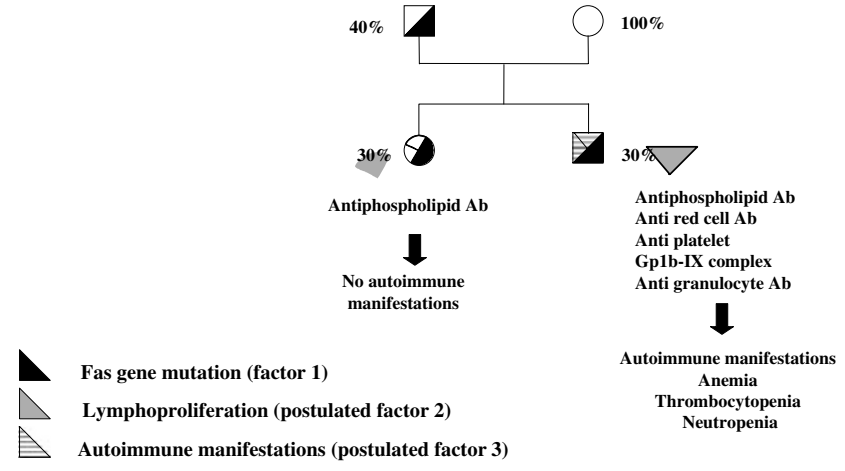

Figure 4 Genetic events leading to lymphoproliferative syndrome and autoimmunity. In this family, the father is a healthy heterozygous Fas mutation carrier (black symbol). Both children received the mutated paternal allele and developed a typical ALPS la (black and gray symbol). The boy developed severe autoimmunity (hatched symbol) whereas the girl did not exhibit any autoimmune manifestations. Percentages indicate the sensitivity to Fas-induced apoptosis as compared to controls

\section{Conclusions}

The recent unraveling of the molecular events leading to the ALPS syndromes or humans has brought a significant insight into the understanding of the function of the FasL/Fas pathway in lymphocyte homeostasis. More questions are now raised about the possible connections between apoptosis pathways, proliferation and autoimmunity, the three components of ALPS.

\section{References}

1. Ashkenazi A and Dixit VM (1998) Death receptors: signaling and modulation. Science 281: 1305-1308

2. Locksley RM, Killeen N and Lenardo MJ (2001) The TNF and TNF receptor superfamilies: integrating mammalian biology. Cell 104: 487-501

3. Algeciras-Schimnich A, Shen L, Barnhart BC, Murmann AE, Burkhardt JK and Peter ME (2002) Molecular ordering of the initial signaling events of CD95. Mol. Cell Biol. 22: 207-220

4. Thornberry NA and Lazebnik Y (1998) Caspases: enemies within. Science 281: $1312-1316$

5. Yang X, Khosravi-Far R and Baltimore D (1997) Daxx, a novel Fas-binding protein that activates JNK and Apoptosis. Cell 89: 1067-1076

6. Stanger B, Leder P, Lee T, Kim E and Seed B (1995) RIP: a novel protein containing a death domain that interacts with fas/APO-1(CD95) in yeast and causes cell death. Cell 81: 513-523

7. Chu K, Niu X and Williams LT (1995) A Fas-associated protein factor FAF1 potentiates Fas-mediated apoptosis. Proc. Natl. Acad. Sci. USA 1995: 11894-11898

8. Siegel RM, Frederiksen JK, Zacharias DA, Chan FK, Johnson M, Lynch D, Tsien RY and Lenardo MJ (2000) Fas preassociation required for apoptosis signaling and dominant inhibition by pathogenic mutations. Science 288 : 2354-2357

9. Thome M and Tschopp J (2001) Regulation of lymphocyte proliferation and death by FLIP. Nat. Rev. Immunol. 1: 50-58

10. Siegel RM, Chan FK, Chun HJ and Lenardo MJ (2000) The multifaceted role of Fas signaling in immune cell homeostasis and autoimmunity. Nat. Immunol. 1: 469-474

11. Canale VC and Smith CH (1967) Chronic lymphadenopathy simulating malignant lymphoma. J. Pediatr. 70: 891-899

12. Nagata S, and Golstein P (1995) The Fas death factor. Science 267: 14491455 
13. Nagata S, and Suda T (1995) Fas and Fas ligand: Ipr and gld mutations. Immunol. Today 16: 39-43

14. Nagata S (1997) Apoptosis by death factor [Review]. Cell 88: 355-365

15. Rieux-Laucat F, Le Deist F, Hivroz C, Roberts I, Debatin K, Fischer A and de Villartay J (1995) Mutations in fas associated with human lymphoproliferative syndrome and autoimmunity. Science 268: 1347-1349

16. Fisher GH, Rosenberg FJ, Straus SE, Dale JK, Middleton LA, Lin AY, Strober W, Lenardo MJ and Puck JM (1995) Dominant interfering Fas gene mutations impair apoptosis in a human autoimmune lymphoproliferative syndrome. Cell 935-946

17. Drappa J, Vaishnaw AK, Sullivan KE, Chu J-L and Elkon KB (1996) Fas gene mutations in the Canale-Smith syndrome an inherited lymphoproliferative disorder associated with autoimmunity. N. Engl. J. Med. 335: 1643-1649

18. Le Deist F, Emile JF, Rieux-Laucat F, Benkerrou M, Roberts I, Brousse $N$ and Fischer A (1996) Clinical, immunological, and pathological consequences of Fas-deficient conditions. Lancet 348: 719-723

19. Benkerrou M, Le Deist F, de Villartay J, Caillat-Zuckman S, Rieux-Laucat F, Jabado N, Cavazzana-Calvo M and Fischer A (1997) Correction of fas (CD95) deficiency by haploidentical bone marrow transplantation. Eur. J. Immunol. 27: 2043-2047

20. Kasahara Y, Wada T, Niida Y, Yachie A, Seki H, Ishida Y, Sakai T, Koizumi F, Koizumi S, Miyawaki T and Taniguchi N (1998) Novel Fas (CD95/APO-1) mutations in infants with a lymphoproliferative disorder. Int. Immunol. 10: 195-202

21. van der Burg M, de Groot R, Comans-Bitter WM, den Hollander JC, Hooijkaas $\mathrm{H}$, Neijens HJ, Berger RM, Oranje AP, Langerak AW and van Dongen JJ (2000) Autoimmune lymphoproliferative syndrome (ALPS) in a child from consanguineous parents: a dominant or recessive disease? Pediatr. Res. 47: $336-343$

22. Wang J, Zheng L, Lobito A, Chan FK, Dale J, Sneller M, Yao X, Puck JM, Straus SE and Lenardo MJ (1999) Inherited human caspase 10 mutations underlie defective lymphocyte and dendritic cell apoptosis in autoimmune lymphoproliferative syndrome type II. Cell 98: 47-58

23. Rieux-Laucat F, Blachere S, Danielan S, De Villartay JP, Oleastro M, Solary E, Bader-Meunier B, Arkwright P, Pondare C, Bernaudin F, Chapel H, Nielsen S, Berrah M, Fischer A and Le Deist F (1999) Lymphoproliferative syndrome with autoimmunity: A possible genetic basis for dominant expression of the clinical manifestations. Blood 94: 2575-2582

24. Infante AJ, Britton HA, DeNapoli T, Middelton LA, Lenardo MJ, Jackson CE, Wang J, Fleisher T, Straus SE and Puck JM (1998) The clinical spectrum in a large kindred with autoimmune lymphoproliferative syndrome caused by a Fas mutation that impairs lymphocyte apoptosis. J. Pediatr. 133: 629-633

25. Jackson CE, Fischer RE, Hsu AP, Anderson SM, Choi Y, Wang J, Dale JK, Fleisher TA, Middelton LA, Sneller MC, Lenardo MJ, Straus SE and Puck JM (1999) Autoimmune lymphoproliferative syndrome with defective Fas: genotype influences penetrance. Am. J. Hum. Genet. 64: 1002-1014

26. Martin DA, Combadiere B, Hornung F, Jiang D, McFarland H, Siegel R, Trageser C, Wang J, Zheng L and Lenardo MJ (1998) Molecular genetic studies in lymphocyte apoptosis and human autoimmunity. Novartis Found. Symp. 215: 73-82; discussion 82-91

27. Vaishnaw AK, Orlinick JR, Chu JL, Krammer PH, Chao MV and Elkon KB (1999) The molecular basis for apoptotic defects in patients with CD95 (Fas/ Apo-1) mutations. J. Clin. Invest. 103: 355-363

28. Sneller MC, Wang J, Dale JK, Strober W, Middelton LA, Choi YN, Fleisher TA, Lim MS, Jaffe ES, Puck JM, Lenardo MJ and Straus SE (1997) Clinical, immunologic, and genetic features of an autoimmune lymphoproliferative syndrome associated with abnormal lymphocyte apoptosis. Blood 89: $1341-1348$

29. Pensati L, Costanzo A, lanni A, Accapezzato D, lorio R, Natoli G, Nisini R, Almerighi C, Balsano C, Vajro P, Vegnente A and Levrero M (1997) Fas/Apo1 mutations and autoimmune lymphoproliferative syndrome in a patient with type 2 autoimmune hepatitis. Gastroenterology 113: 1384-1389

30. DeFranco S, Bonissoni S, Cerutti F, Bona G, Bottarel F, Cadario F, Brusco A, Loffredo G, Rabbone I, Corrias A, Pignata C, Ramenghi U and Dianzani U (2001) Defective function of Fas in patients with type 1 diabetes associated with other autoimmune diseases. Diabetes 50: 483-488

31. Straus SE, Sneller M, Lenardo MJ, Puck JM and Strober W (1999) An inherited disorder of lymphocyte apoptosis: the autoimmune lymphoproliferative syndrome. Ann. Intern. Med. 130: 591-601
32. Straus SE, Jaffe ES, Puck JM, Dale JK, Elkon KB, Rosen-Wolff A, Peters AM, Sneller MC, Hallahan CW, Wang J, Fischer RE, Jackson CM, Lin AY, Baumler C, Siegert E, Marx A, Vaishnaw AK, Grodzicky T, Fleisher TA and Lenardo MJ (2001) The development of lymphomas in families with autoimmune lymphoproliferative syndrome with germline Fas mutations and defective lymphocyte apoptosis. Blood 98: 194-200

33. Boulanger E, Rieux-Laucat F, Picard C, Legall M, Sigaux F, Clauvel JP, Oksenhendler E, Le Deist F and Meignin V (2001) Diffuse large B-cell nonHodgkin's lymphoma in a patient with autoimmune lymphoproliferative syndrome. Br. J. Haematol. 113: 432-434

34. Landowski TH, Qu N, Buyuksal I, Painter JS and Dalton WS (1997) Mutations in the Fas antigen in patients with multiple myeloma. Blood 90: 4266-4270

35. Beltinger C, Kurz E, Bohler T, Schrappe M, Ludwig WD and Debatin KM (1998). CD95 (APO-1/Fas) mutations in childhood T-lineage acute lymphoblastic leukemia. Blood 91: 3943-3951

36. Bleesing JJ, Brown MR, Straus SE, Dale JK, Siegel RM, Johnson M, Lenardo MJ, Puck JM and Fleisher TA (2001) Immunophenotypic profiles in families with autoimmune lymphoproliferative syndrome. Blood 98: 2466-2473

37. Lopatin U, Yao X, Williams RK, Bleesing JJ, Dale JK, Wong D, Teruya-Feldstein J, Fritz S, Morrow MR, Fuss I, Sneller MC, Raffeld M, Fleisher TA, Puck JM, Strober W, Jaffe ES, and Straus SE (2001) Increases in circulating and lymphoid tissue interleukin-10 in autoimmune lymphoproliferative syndrome are associated with disease expression. Blood 97: $3161-3170$

38. Fuss IJ, Strober W, Dale JK, Fritz S, Pearlstein GR, Puck JM, Lenardo MJ and Straus SE (1997) Characteristic T helper 2 T cell cytokine abnormalities in autoimmune lymphoproliferative syndrome, a syndrome marked by defective apoptosis and humoral autoimmunity. J. Immunol. 158: 1912-1918

39. Wu JG, Wilson J, He J, Xiang LB, Schur PH and Mountz JD (1996) Fas ligand mutation in a patient with systemic lupus erythematosus and lymphoproliferative disease. J. Clin. Invest. 98: 1107-1113

40. Itoh N, and Nagata S (1993) A novel protein domain required for apoptosis. Mutational analysis of human Fas antigen. J. Biol. Chem. 268: 10932-10937

41. Bettinardi A, Brugnoni D, Quiros-Roldan E, Malagoli A, La Grutta S, Correra A and Notarangelo LD (1997) Missense mutations in the Fas gene resulting in autoimmune lymphoproliferative syndrome: a molecular and immunological analysis. Blood 89: 902-909

42. Lim MS, Straus SE, Dale JK, Fleisher TA, Stetler-Stevenson M, Strober W, Sneller MC, Puck JM, Lenardo MJ, Elenitoba-Johnson KS, Lin AY, Raffeld M and Jaffe ES (1998) Pathological findings in human autoimmune lymphoproliferative syndrome. Am. J. Pathol. 153: 1541-1550

43. Aspinall Al, Pinto A, Auer IA, Bridges P, Luider J, Dimnik L, Patel KD, Jorgenson K and Woodman RC (1999) Identification of new Fas mutations in a patient with autoimmune lymphoproliferative syndrome (ALPS) and eosinophilia. Blood Cells Mol. Dis. 25: 227-238

44. Avila NA, Dwyer AJ, Dale JK, Lopatin UA, Sneller MC, Jaffe ES, Puck JM and Straus SE (1999) Autoimmune lymphoproliferative syndrome: a syndrome associated with inherited genetic defects that impair lymphocytic apoptosis CT and US features. Radiology 212: 257-263

45. Arkwright PD, Rieux-Laucat F, Le Deist F, Stevens RF, Angus B and Cant AJ (2000) Cytomegalovirus infection in infants with autoimmune lymphoproliferative syndrome (ALPS). Clin. Exp. Immunol. 121: 353-357

46. Bader-Meunier B, Rieux-Laucat $F$, Croisille $L$, Yvart J, Mielot $F$, Dommergues JP, Ledeist $F$ and Tchernia $G$ (2000) Dyserythropoiesis associated with a fasdeficient condition in childhood. Br. J. Haematol. 108: 300-304

47. Shenoy S, Arnold S and Chatila T (2000) Response to steroid therapy in autism secondary to autoimmune lymphoproliferative syndrome. J. Pediatr. 136: $682-687$

48. Martin DA, Zheng L, Siegel RM, Huang B, Fisher GH, Wang J, Jackson CE, Puck JM, Dale J, Straus SE, Peter ME, Krammer PH, Fesik S and Lenardo MJ (1999). Defective CD95/APO-1/Fas signal complex formation in the human autoimmune lymphoproliferative syndrome, type la. Proc. Natl. Acad. Sci. USA 96: 4552-4557

49. Adachi M, Suematsu S, Kondo T, Ogasawara J, Tanaka T, Yoshida N and Nagata S (1995) Targeted mutation in the Fas gene causes hyperplasia in peripheral lymphoid organs and liver. Nat. Genet. 11: 294-300 
50. Kimura M, Ikeda H, Katagiri T and Matsuzawa A (1991) Characterization of lymphoproliferation induced by interactions between Iprcg and gld genes. Cell Immunol. 134: 359-369

51. Wang J, Chun HJ, Wong W, Spencer DM and Lenardo MJ (2001) Caspase-10 is an initiator caspase in death receptor signaling. Proc. Natl. Acad. Sci. USA 98: 13884-13888

52. Kischkel FC, Lawrence DA, Tinel A, LeBlanc H, Virmani A, Schow P, Gazdar A Blenis J, Arnott D and Ashkenazi A (2001) Death receptor recruitment of endogenous caspase-10 and apoptosis initiation in the absence of caspase-8. J. Biol. Chem. 276: 46639-46646

53. Inguli E, Mondino A, Khoruts A and Jenkins MK (1998) In vivo detection of dendritic cell antigen presentation to CD4 $T$ cells. J. Exp. Med. 185: 2133-2141

54. Chun HJ, Zheng L, Ahmad M, Wang J, Speirs CK, Siegel RM, Dale JK, Puck J, Davis J, Hall CG, Skoda-Smith S, Atkinson TP, Straus SE and Lenardo MJ (2002) Pleiotropic defects in lymphocyte activation caused by caspase-8 mutations lead to human immunodeficiency. Nature 419: 395-399

55. Zhang J, Cado D, Chen A, Kabra NH and Winoto A (1998) Fas-mediated apoptosis and activation-induced $\mathrm{T}$-cell proliferation are defective in mice lacking FADD/Mort1. Nature 392: 296-300
56. Liu J, Na S, Glasebrook A, Fox N, Solenberg PJ, Zhang Q, Song HY and Yang DD (2001) Enhanced CD4+ T cell proliferation and Th2 cytokine production in DR6-deficient mice. Immunity 15: 23-34

57. Zhao H, Yan M, Wang H, Erickson S, Grewal IS and Dixit VM (2001) Impaired c-Jun amino terminal kinase activity and $\mathrm{T}$ cell differentiation in death receptor 6-deficient mice. J. Exp. Med. 194: 1441-1448

58. Wang EC, Thern A, Denzel A, Kitson J, Farrow SN and Owen MJ (2001) DR3 regulates negative selection during thymocyte development. Mol. Cell Biol. 21: 3451-3461

59. Strauss SE, Sneller M, Lenardo MJ, Puck JM and Strober W (1999) The autoimmune lymphoproliferative syndrome. Ann. Int. Med. 130: 591-601

60. Rathmell JC, Cooke MP, Ho WY, Grein J, Townsend SE, Davis MM and Goodnow CC (1995) CD95 (Fas)-dependent elimination of self-reactive B cells upon interaction with CD4+ T cells. Nature 376: 181-184

61. Singer GG and Abbas AK (1994) The fas antigen is involved in peripheral but not thymic deletion of $\mathrm{T}$ lymphocytes in $\mathrm{T}$ cell receptor transgenic mice. Immunity 1: 365-371

62. Vidal S, Kono DH and Theofilopoulos AN (1998) Loci predisposing to autoimmunity in MRL-Fas Ipr and C57BL/6-Faslpr mice. J. Clin. Invest. 101: 696-702 